\section{Palliative sedation: Improvement of guidelines necessary, but not sufficient}

Editor - We would like to congratulate Seymour et al. ${ }^{1}$ for their insights gained by the qualitative study on the controversial practice of continuous sedation until death in three European countries. Based on our clinical experience and recent research ${ }^{2,3}$ on palliative sedation therapy (PST), we would like to comment on some practical as well as conceptual issues raised in this publication.

First, the authors state that the practice of PST varies 'in spite of clear guidelines' (p. 1). ${ }^{1}$ However, in line with the findings of our recently conducted systematic review of published guidelines on PST, we argue that there is considerable variation on the level of guidelines. ${ }^{2,3}$ The guidelines differ regarding the concepts of PST, the recommendations on indication and decision-making for $\mathrm{PST}^{2}$ and regarding medical issues such as recommendations on type of drugs, mode of titration and monitoring. ${ }^{3}$ In addition, the assessment of the quality of the guidelines according to the criteria defined by the AGREE consortium ${ }^{4}$ indicates that there is currently no guideline on PST published in peer-reviewed journals which reaches high marks in all quality domains. ${ }^{3}$ Against this background, we argue that the development of future guidelines should follow the established quality criteria as far as these can be applied to this topic to support healthcare professionals with regards to decisions around PST. However, in light of the views and experiences elicited by Seymour et al., we question whether good guidelines alone will be sufficient and suggest that they need to be supplemented by measures to facilitate and enhance reflection about individual judgements and decisions based on these guidelines - such as case conferences or, in particularly challenging cases, clinical ethics consultation.

Second, the interesting findings on differing perceptions of continuous palliative sedation as 'alternative' to euthanasia (i.e. killing on request) raise important questions how far we can understand these two measures as - although clearly different - 'alternatives'. Most obviously, the situation for healthcare professionals in Belgium and the Netherlands differs from the United Kingdom and most other countries in the world insofar as in the former two countries, killing on request or assisted suicide is a 'legal alternative'. However, continuous sedation until death and killing on request can also be understood as 'alternatives' with reference to different values held by individual patients. While for some patients the idea of dying while sleeping (as in continuous sedation) is in line with their ideal of a good death, there are also patients who would prefer to end their life at one point in time without any compromise to their level of consciousness (as in killing on request or assisted suicide). Notwithstanding to both aforementioned understandings of continuous sedation until death as 'alternative' to killing on request, it should be emphasised that PST per se is not an ethically unproblematic 'alternative'. Rather and much in line with the analysis of Seymour et al., we suggest that the three following clinical-ethical challenges of PST warrant further exploration: (1) a sound approach to professional decision-making about PST as an indicated treatment option, (2) the involvement of patients or their representatives in any part of the decision-making process which needs to be informed by the patient's values and preferences and (3) a clear distinction of decisions about PST and accompanying treatment such as parenteral hydration. Against this background, we suggest that further qualitative research on differences of practices of PST within countries and in-depth exploration of factors associated with differing practices (e.g. differing concepts of continuous sedation or different notions of a good end of life) can contribute to further critical reflection about current PST practice. In addition, such research may also point out ways to further improve standards of care in this clinically and ethically challenging domain of end-of-life care.

\section{Declaration of conflicting interests}

We declare that we have no conflicts of interest.

\section{Funding}

This research received no specific grant from any funding agency in the public, commercial or not-for-profit sectors.

\section{References}

1. Seymour J, Rietjens J, Bruinsma S, et al.; on behalf of the UNBIASED consortium. Using continuous sedation until death for cancer patients: a qualitative interview study of physicians' and nurses' practice in three European countries. Palliat Med. Epub ahead of print 25 July 2014. DOI: $10.1177 / 0269216314543319$. 
2. Schildmann EK and Schildmann J. Palliative sedation therapy: a systematic literature review and critical appraisal of available guidance on indication and decision making. $J$ Palliat Med 2014; 17(5): 601-611.

3. Schildmann EK, Schildmann J and Kiesewetter I. Medication and monitoring in palliative sedation therapy: a systematic review and quality assessment of published guidelines. $J$ Pain Symptom Manage. Epub ahead of print 18 September 2014. DOI: 10.1016/j.jpainsymman.2014.08.013.

4. Agree Next Steps Consortium. The AGREE II instrument (Electronic version), http://www.agreetrust.org (2009, accessed 25 September 2014).
Eva K Schildmann ${ }^{1}$, Claudia Bausewein ${ }^{1}$

and Jan Schildmann ${ }^{2}$

${ }^{1}$ Department of Palliative Medicine, Munich University Hospital, Munich, Germany

2Institute for Medical Ethics and History of Medicine, Ruhr-University Bochum, Bochum, Germany

\section{Corresponding author:}

Eva K Schildmann, Department of Palliative Medicine, Munich University Hospital, Marchioninistr. 15, 81377 Munich, Germany. Email: Eva.schildmann@med.uni-muenchen.de 